\title{
A personal perspective on Raghu Varadhan's role in the development of Stochastic Analysis
}

\author{
Terry Lyons, University of Oxford
}




\section{Contents}

I Professor SRS Varadhan 1

0.1 A Great Day for the Coin Flippers . . . . . . . . . . . . . 3

0.2 Stochastic Analysis . . . . . . . . . . . . . . . 3

0.3 Varadhan . . . . . . . . . . . . . . . . 5

0.4 Independent or Uncorrelated $\ldots \ldots \ldots \ldots \ldots$

$0.4 .1 \quad$ A Simple Example . . . . . . . . . . . . . . . 7

0.5 Diffusion Equations and PDE . . . . . . . . . . . . 9

0.5.1 Elliptic PDEs in Non-Divergence Form with Continuous

Elliptic Coefficients - Uniqueness of Solution . . . . . . 11

0.5.2 First-Order PDEs and Large Deviations . . . . . . . . . . 13

0.6 The Support Theorem-Understanding the Itô Differential Equa-

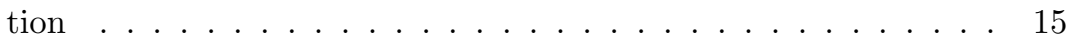

0.7 The Donsker-Varadhan Theory of Large Deviations . . . . . . 20

0.8 Hydrodynamical Limits, Interacting Particles and Other Questions 26

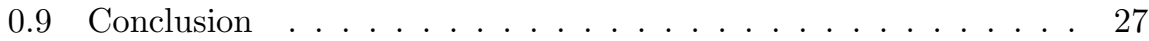




\section{Part I}

\section{Professor SRS Varadhan}





\subsection{A Great Day for the Coin Flippers}

I know Rahgu Varadhan professionally but not personally - that is to say we have attended some of the same conferences and Oberwolfach meetings, and even the odd meal while waiting for trains home. Still, it is obvious to me, and I am sure to anyone else who comes close, that he is a person of great humanity who generates warmth and humour whenever he is in the room. A few months after the award of Fields Medals to Werner, Okounkov and Tao in Madrid, Varadhan and I were both in a group of mathematicians talking about the event. I remember clearly Varadhan's concise summary of the business as "A great day for the coin flippers". It certainly was: all three used probability in their ground-breaking work and, for the first two, Stochastic Analysis has been a decisive part of their mathematical toolbox. We were all excited that stochastic ideas were having such a substantial effect across areas as far apart as conformal field theory, geometry and number theory. We were also delighted that these achievements were recognized. To me, Varadhan's remark seemed to capture his modesty and humour rather well. Surely it was another excellent day for the coin flippers when Varadhan was awarded the Abel Prize.

\subsection{Stochastic Analysis}

Stochastic Analysis is an area of mathematics that, in a little over 60 years, has grown from almost nothing to a significant field. It has importance for its intrinsic interest and for its contributions to other foundational "pure" areas of mathematics, as well as for its contributions to the applications of mathematics. These applications seem to be on an enormous and expanding scale, spanning engineering and climate modelling. It is totally clear to those that work in Stochastic Analysis that Varadhan has shaped the subject in hugely significant ways, both in his personal contributions - in the sense of direction he has givenand in the guidance he has provided to his many outstanding colleagues and students.

I am no historian, and my remarks assigning historical credit should be treated with caution. Given this caveat, I would like to try to place Varadhan's contributions in context by mentioning a few of the landmark contributions that have shaped the Stochastic Analysis scenery. At least for me, the first wave in the development of Stochastic Analysis is associated with a Russian, a Frenchman, a Japanese man, and an American. The Russian is Kolmogorov, a tower of 20th century mathematics who gave us a rigorous framework for the mathematical study of probability. The Frenchman is Lévy, who made a detailed mathematical study of Brownian motion and Lévy processes. The Japanese man is Itô, who extended differential calculus to the Brownian case and so gave us stochastic calculus. The American is Doob, who gave us the ubiquitous martingale with its optional stopping theorem and up-crossing lemma, and the tools required to provide mathematical confirmation that, in a fair world, there are no free lunches. It is impossible to convey more than a few hints as to the 
full significance of each of these mathematicians' contributions.

Kolmogorov's 66 page monograph, which in 1933 set out a rigorous framework of probability, and his strong law of large numbers, form essential building blocks allowing the rigorous mathematical study of infinite-dimensional probabilistic objects, such as Brownian motion (as studied by Lévy), to flourish. Modern mobile phone technology depends on the strong law of large numbers to separate the transmissions from different phones and allow robust transmission in the context of noise and interference.

Newtonian calculus and differential equations are the classical tools for expressing interactions between evolving systems. Itô's stochastic calculus (1942) extends the remit of differential equations to systems driven by random processes such as Brownian motion. Itô's theory gave a direct connection between probability theory and a wide class of second order parabolic partial differential equations. It also provided the framework for many fundamental applications, for example, the continuous time Kalman-Bucy filter which revolutionized the field of estimation and was a major breakthrough in guidance technology (the lunar landing of Apollo is a well documented example). Practical application in non-linear settings requires the construction of numerical approximations to the solutions to certain non-linear PDEs (partial differential equations). Stochastic filters are used in almost all modern military and commercial control systems.

Doob took ideas that were well known in function theory about the behaviour of harmonic functions on the boundary of the disc (Fatou's theorem, Littlewood's theorem) and showed how they had probabilistic parallels. He introduced martingales, established optional stopping and up-crossing lemmas, quantified the oscillatory behaviour, and established convergence properties. In effect, he explained how to generalise the notion of a parabolic PDE to the context of functions defined on spaces of paths. It was a remarkable achievement, demonstrating the enormous power a well chosen abstraction can have.

There is, and will remain, a substantial demand for financial intermediaries, who are able to supply trustworthy and economically priced products, that allow individuals and businesses to insure against financial risks in areas of their business where they have limited expertise and control. Financial intermediaries, who supply these products, must hedge their liabilities, and in general, this hedge will be dynamic and change from day to day, so as to ensure that, at the time an insurance policy matures, the intermediary has the appropriate resources to pay any claim against it.

The correction term (Itô's formula) to Newtonian Calculus to account for the volatility of market prices is at the heart of this hedging process. The provision of these basic insurance activities has become standard over the last 15 years, increasing transparency and forcing margins down. The need for insurance against interest rate and currency fluctuations on a huge scale will not disappear as a result of the current financial storm although one might expect providers to increase their margins somewhat.

Of course, these four mathematicians were not alone (Chung, Dynkin, Kintchine, McKean, Malliavin, and Meyer were some of the others). Nor had the full significance of their contributions been appreciated when Varadhan began 
to make his own critical interventions.

None the less, it was clear (to those in the area) that Stochastic Analysis had become a set of tools and techniques that could give insight into really quite high-dimensional systems. If one models the evolution of a population, one might use a parabolic PDE to describe the mean local density or intensity of the population as it evolved. Probability can inject additional insight into such deterministic systems. Stochastic analysts have developed tools that model the behaviour of the underlying stochastic population as well as its mean behaviour. This extra step requires more effort and genuinely probabilistic tools; but the value of this distinction, emphasizing the importance of sample paths, provides a clear advantage. In settings, such as control or modelling financial markets, where one has to understand how to interact with and respond to the actual - if uncertain - evolution of the world, the distinction is decisive. Average behaviour is interesting but is often far from the whole story.

Probabilists are not unhappy when the their systems are so large and internally homogeneous that on large scales they behave as if they were deterministic. They sometimes call this the fluid or hydrodynamic limit, even though fluids frequently retain very random behaviour on normal as well as microscopic scales!

Varadhan, his collaborators, and his students have made quite fundamental contributions linking the theory of probability to the theory of PDEs, in capturing the information about systems that PDEs miss (large deviations), and in proving that interesting microscopic random systems do indeed have hydrodynamic limiting behaviour. These contributions place him at the core of these developments. I am sure that in his modesty, Varadhan would be the first to note that he is not alone in making fundamental contributions to Stochastic Analysis (for example the work of Friedlin and Ventcell was around the same time and tackles similar issues to some of Varadhan's work), but I am sure that everyone in the field was delighted when Varadhan was awarded the Abel Prize.

\section{$0.3 \quad$ Varadhan}

Varadhan's contributions are plentiful, original, beautiful, and surprising. They have had strategic significance in many different directions, so that no-one except perhaps Varadhan could be authoritative on them all, and I doubt if Varadhan could have guessed the range of ways they would be used. I will not (and cannot) attempt a comprehensive survey. I agreed to write this article because I thought it was a great opportunity to express my (inevitably limited) understanding for some of the ways these results have had an impact on the development of Stochastic Analysis, and to explain, through some of Varadhan's striking work, why I think Stochastic Analysis remains an exciting cornerstone of modern mathematics.

Amongst Varadhan's contributions to the development of probability and analysis, I should, at the very least, draw attention to the creation and development of the theory of Large Deviations with Donsker, upon which so much of our understanding of stochastic systems depends. I must also mention the de- 
velopment, alone and with Stroock, of the martingale method for characterising diffusions. This work, with its roots firmly in the probability theory developed by Doob et al., showed the power of the new methods. In a deep piece of work, Stroock and Varadhan produced the first truly satisfactory treatment of elliptic second order parabolic PDEs (in non-divergence form) with continuous coefficients; through this work they demonstrated the power of the new technologies. I should also draw attention to the beautiful work on short time behaviour of diffusions, and mention Varadhan's major foundational contributions to the theory of interacting particle systems and to the development of a theory of hydrodynamic limits for these systems. Varadhan's seminal work with Lions and Papanicolou on the homogenization of the Hamilton-Jacobi equation also has to be on any list, although, unfortunately, space and time mean that I cannot include everything I might wish.

\subsection{Independent or Uncorrelated}

It might seem like a contradiction, but rare events happen. They can happen frequently, and the nature of the rare events that happen can have an impact in significant ways on the systems around us. I drive a car. I only do this because I believe it is really unlikely that I will have a serious accident - I see it as a rare event. On the other hand, there are many millions of drivers, and looked at across the whole population it is very likely that there will be several car accidents in a single day, and that, sadly, some will be very serious. Car accidents are rare, but they will happen because there are lots of cars.

Now, suppose that there are two classes of accidents: $A$ and $B$. Then asserting that $A$ and $B$ are rare events is essentially saying that $\mathbb{P}(A)$ and $\mathbb{P}(B)$ are both small. However, in this context it is worth appreciating that, in general, rare events are not likely to be equally rare. For example, it may be that $\mathbb{P}(A)=1 / 100$ and $\mathbb{P}(B)=1 / 1000$. In this case it is trivial to see that, conditional on one of these two events occurring, then it is about 10 times more likely to be an event of type $A$ than of type $B$ which occurs. It does not take much imagination to realize that in large populations which interact in nonlinear ways, it becomes very important to understand the rare events and their relative probability. Rare events can play a key role in determining the function of the entire system.

The theory of Large Deviations provides a systematic mathematical framework for describing and estimating the probabilities of certain rare or exceptional events and predicting their consequences.

Large Deviation theory is a crucial counterbalance to the much better known Central Limit Theorem. The latter remarkable result considers the accumulated effect of repeated independent events. It is a remarkable result that says that the sample mean of the sum of independent and identically distributed variables $X_{i}$ with finite variance and mean zero has a distribution that is very close to 
Gaussian; if $A$ is open, then:

$$
\mathbb{P}\left(\frac{\sum_{i=1}^{N} X_{i}}{\sqrt{N}} \in A\right)=\int_{A} \frac{e^{-\frac{x \sigma^{-1} x}{2}} d x}{|\sigma|^{1 / 2}(2 \pi)^{d / 2}}+o(1)
$$

where $\sigma$ is the covariance of $X$. The strength and the implications of the Central Limit Theorem cannot be underestimated. It is a hugely valuable theorem, and it can be generalised to random paths.

The limiting distribution is simple and easily described and is entirely determined by the correlations of the underlying incremental event $X$. However, the central limit theorem only refers to the likely events. It says that, for the vast majority of the time, the sample mean behaves as if it was a multivariate normally distributed random variable; the covariance $\sigma$ of $X$ is all you have to know to understand the distribution of these typical fluctuations.

However, the Central Limit Theorem has nothing to say about rare events, those that happen on the scale of probabilities $e^{-c N}$ or for the probabilities of fluctuations of the sample mean that are $o(1)$, say. These rare events often have a rich behaviour, and at the same time they often play a decisive role in the behaviour of complex systems.

\subsubsection{A Simple Example}

Let $X_{i} \in \mathbb{Z}^{d}$ be a sequence of independent, identically distributed, random outcomes with mean zero and finite support. Suppose the symmetric quadratic form (known as the covariance):

$$
C(u, v):=\mathbb{E}((u . X)(v \cdot X))
$$

is finite and non-degenerate, with inverse $g$. Consider the random walk on $\mathbb{Z}^{d}$ whose transition is $X$. That is to say, let $S_{i}$ represented the accumulation of these events

$$
S_{n}=\sum_{j=0}^{n} X_{j}
$$

It is a basic problem to understand the macroscopic behaviour of the process $S_{n}$, and from a certain point of view Donsker's Functional Central Limit Theorem provides a very complete answer (see M.D. Donsker, "Justification and extension of Doob's heuristic approach to the Kolmogorov-Smirnov theorems", Annals of Mathematical Statistics, 23:277-281, 1952). The process $B_{t}^{(n)}:=g^{1 / 2} n^{-1 / 2} S_{\lfloor n t\rfloor}$ is a very close approximation to Brownian motion; more precisely, its law as a measure on paths converges in the weak topology to Wiener measure.

It is standard practise in many areas of modelling where one accepts that there is a natural independence over disjoint time horizons to measure short term correlations, and then to model the process over longer time horizons using a renormalized Brownian motion. Donsker's theorem provides a limited 
justification for this. But there are dangers and, in the context of rare events, it is simply unsound.

For example, a credit agency might wish to assess the probability that a product could fail. They might believe it was safe because, although each component asset was risky, they believed that the probability of a large number of the components failing at one time was very small. This would be the case, if the components behaved independently. However, it would be inordinately difficult to create an empirical test for independece of a large number of these components. However, they may well be able to carry out accurate empirical tests which justify assuming that the values of the components are uncorrelated. It might also be reasonable to assume that the incremental behaviour of these values is independent from one time step to another. Is it reasonable for the agency to use a $d$-dimensional Brownian motion to model the evolution of these assets? In general, there is a scale on which the answer is "yes", but there is a larger scale on which this is a profoundly dangerous thing to do. The large deviations for the actual process might be quite different to the large deviations of a Brownian motion. In general, assuming that a number of events are independent leads to unrealistically small probabilities of rare events. There might well be strong empirical evidence that there is a 1 in $10^{4}$ probability that two of the assets lose their value, and the assumption of independence would set the probability of three losing their value at about 1 part in $10^{6}$. We can see how reliable this approach is by considering an example. Suppose that $X$ takes one of four values

$$
\{1,1,1\},\{1,-1,-1\},\{-1,1,-1\},\{-1,-1,1\}
$$

and each is equally likely. Then it is easy to check that $X$ has the same covariance as the increment of a 3 -dimensional Brownian motion over a unit time step. Let $X_{j}$ be independent with distribution equal to $X$ and set

$$
S_{n}=\sum_{j=0}^{n} X_{j} .
$$

The central limit theorem suggests that $S_{n}$ should behave like a Brownian motion and the coordinates should be independent. We can see that this is not true over large scales. Name the coordinate components of $S_{n}$

$$
S_{n}=\left(a_{n}, b_{n}, c_{n}\right) .
$$

What happens if $a_{n}$ and $b_{n}$ are at least $n-m$ ? It must be the case that

$$
\left|\left\{j \mid X_{j}=\{1,1,1\}\right\}\right|>n-2 m
$$

and so $c_{n}>n-2 m$, and for $m<n / 2$ one sees that exceptional behaviour in the first two coordinates forces exceptional behaviour for the third coordinate, contradicting the intuition gained from the fact that the variables are uncorrelated, and the Central Limit Theorem. 
If $\hat{X}_{n}=\frac{1}{n} \sum_{i=1}^{n} X_{i}$ is the sample mean of the $X_{i}$ and $E$ is a Borel set with $0 \notin \bar{E}$ then standard Large Deviations theory gives asymptotic exponential decay rates for the probability that $\hat{X}_{n}$ lies in $E$ :

$$
\begin{aligned}
\liminf \frac{1}{n} \log \mathbb{P}\left(\hat{X}_{n} \in E\right) & \geq \inf _{e \in E^{o}} I(z), \\
I(z) & =\sup _{\lambda \in \mathbb{R}^{d}}(\lambda \cdot x-\log \phi(\lambda)), \\
\phi(\lambda) & =\mathbb{E}\left(e^{\lambda \cdot X_{1}}\right)
\end{aligned}
$$

where $I$ is the Legendre transform of the log of the Laplace transform

$\phi((x, y, z)):=\frac{1}{4}\left(e^{2 x}+e^{2 y}+e^{2 z}+e^{(x+y+z)}\right)(\cosh (x+y+z)-\sinh (x+y+z))$

of the distribution of $X$.

We can compare this with the equivalent upper bound for the case where the increment comes from Brownian motion

$$
\begin{aligned}
\phi(\lambda) & =\mathbb{E}\left(e^{\lambda \cdot B_{1}}\right) \\
& =\exp \left(\frac{1}{2}|\lambda|^{2}\right)
\end{aligned}
$$

the function $\phi$ is quite different; the difference between the two rate functions $I(z)=\sup _{\lambda \in \mathbb{R}^{d}}(\lambda \cdot x-\log \phi(\lambda))$ would show the differences and thus this dichotomy immediately.

Given examples like the above (where the coordinates of the increments are even pair-wise independent, as well as uncorrelated) it is a surprise to this author that, in a wide range of contexts, one observes people quoting incredibly small probabilities for the occurrence of multiple simultaneous coincidences where there cannot be empirical evidence for these probabilities (because they are too small) and where there is no reasonable justification for the joint independence of the events. We can understand that netting makes sense for a few assets in basket of bonds but systematic collapse cannot be ruled out on the basis of empirical evidence. Similar issues arise in criminal law where any claim that the probability of an alternative explanation has extremely small probability, typically obtained by multiplying probabilities, is more likely to be a confirmation of the stochastic illiteracy of the person making the claim than a statement of fact.

Large deviations are not predicted by the co-variance of a system.

\subsection{Diffusion Equations and PDE}

When pollution spreads through soil, when heat moves away from a semiconductor, or when fluid moves through a pipe, one understand that the observed phenomena represent the averaged behaviour of a huge cloud of microscopic diffusive particles. But at least in the first two, and for systems with low Reynolds 
number in the third, the randomness has been so smoothed out on normal scales (by the Central Limit Theorem) that the behaviour has become steady and predictable. Second order elliptic and parabolic PDEs are basic mathematical tools used to model bulk continuum behaviour; these tools are incredibly effective in predicting the behaviour of these systems when they can be applied. However, there are settings where the diffusion is highly inhomogeneous, such as where there are layers of insulation or impervious obstacles distorting this diffusive behaviour even into a lower dimensional flow. In the case of pollution flowing

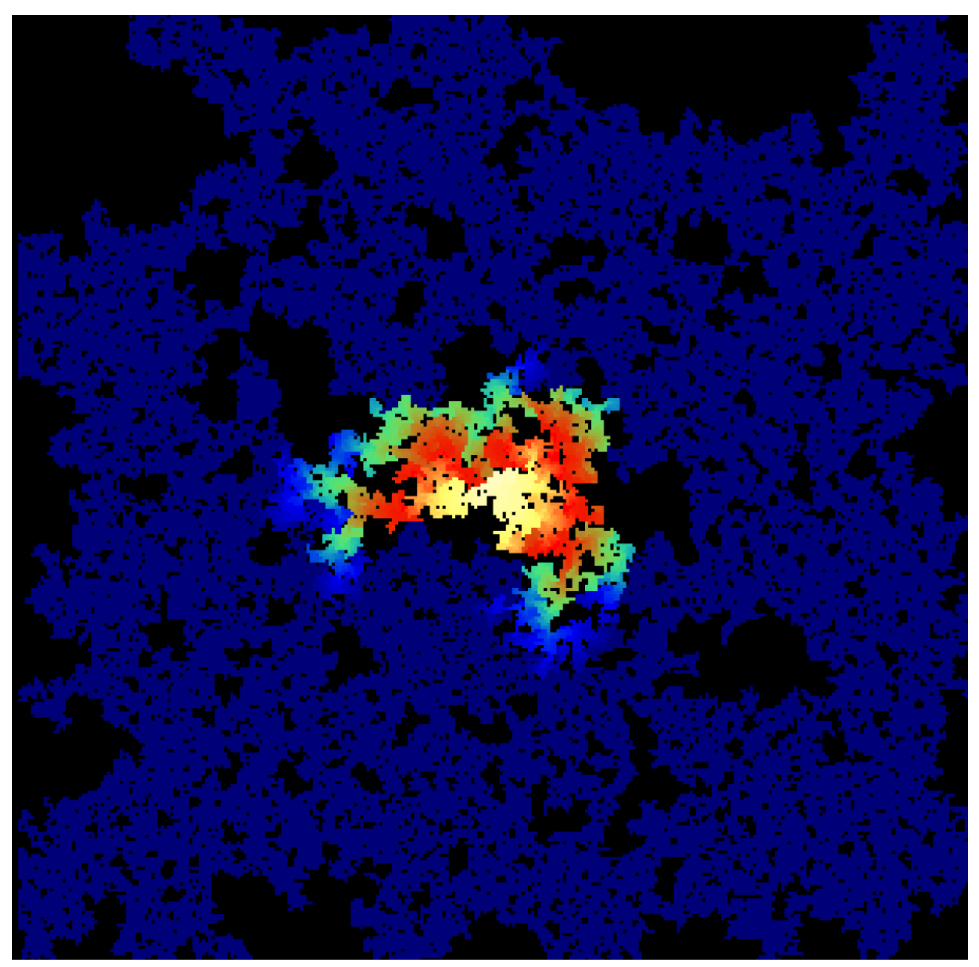

Figure 1: Heat diffusing in a random fractal environment - as simulated by a programme by Martin Barlow.

through a porous media, it is easy to imagine that the diffusion is supported on a set of fractional-dimension. Today we see a division between the Stochastic Analysis methods which are well adapted to describing bulk diffusivity on the widest classes of domain, such as fractal sets, and the PDEs methods which depend on the locally smooth nature of the domain. Progress in understanding this more general setting is steady and many questions which have remained open for considerable periods, for example about uniqueness of diffusions on regular fractals, are now being solved as deeper understandings of things like Harnack inequalities and Dirichlet Forms emerge ${ }^{1}$

\footnotetext{
${ }^{1}$ For a survey see $[1,2]$.
} 
I would not want to suggest that PDE methods are in any sense redundant; they are very effective when they can be used and powerful indicators of how to proceed in more general settings where they cannot be appropriate. For example, it is almost a triviality that, up to scaling, there is only one translation and rotation invariant second order differential operator, and one translation and rotation invariant Markov diffusion on $\mathbb{R}^{n}$. This observation leads one to ask for uniqueness of diffusions respecting the symmetries of a regular fractal. The Bass-Barlow proof of uniqueness for the diffusion on the Sierpinski carpet is very hard!

The flow of understanding is not one way and Varadhan, both alone and with collaborators (particularly with Stroock) demonstrated very clearly in his early work that Stochastic Analysis is a powerful tool for tackling some of the harder and more fundamental questions associated to PDEs. If PDEs model the approximately deterministic macroscopic behaviour of large diffusive populations, then there will be cases where the diffusivity of individual elements in the population remains unchanged by the evolution of neighbouring elements, but in other cases one would expect significant interaction. In this latter case the coefficients in the PDEs modelling the macroscopic diffusion will depend on the solution for their values; a priori they are unknown and the PDEs will be non-linear; and when the environment where the diffusion takes place is rapidly fluctuating on small scales the coefficients will oscillate wildly. Even within the elliptic setting, where the diffusion sees a full ball of neighbours instantly (and is not forced into, for example, cracks or fractals), the need for a priori estimates in the study of non-linear PDEs and the need to understand the large scale behaviour of diffusion in highly fluctuating media each separately justify the extension of the mathematical theory of linear parabolic PDEs to the case where the coefficients of these equations are not smooth. The precise mathematical interpretation of a PDE with less than smooth coefficients remains subtle, even in the elliptic case.

\subsubsection{Elliptic PDEs in Non-Divergence Form with Contin- uous Elliptic Coefficients-Uniqueness of Solution}

A central difficulty is to provide an interpretation of "solution" that is embracing enough to capture the actual diffusive possibilities, while at the same time have an equation that is precise enough that the initial data provided does indeed provide for the unique evolution of the system, and hidden variability does not remain. The approaches split according to whether or not the equations are in divergence form. A key question for the non-divergence form has always been to understand the linear backward operator

$$
L:=\frac{1}{2} \sum_{i, j \leq n} a_{i j}(t, x) \frac{\partial^{2}}{\partial x_{i} \partial x_{j}}+\sum_{i \leq n} b_{i}(x) \frac{\partial}{\partial x_{i}}
$$

where the coefficients are just bounded. Success in such an a priori approach would allow meaning in a rich and straightforward way for the widest classes of 
fully non-linear equations.

A fundamental problem has been to establish the existence and uniqueness of fundamental solutions and the existence of an associated semigroup $P_{t}$. Under smoothness assumptions much was known. Nirenberg [9] had established a maximum principle (uniqueness) if the solution was $C^{2}$, while Dynkin and others had established uniqueness and existence results that went up to the Hölder continuous coefficient case. A very important contribution came from from Tanaka (1964) and independently from Krylov (1966) who established compactness results and showed the existence of (possibly non-unique) semigroups associated to the operator (1) in the continuous coefficient case. However, neither Krylov nor Tanaka proved uniqueness, and uniqueness is every bit as much an issue as existence; without it the standard PDEs associated to (1) would not be well defined.

In a beautifully written pair of papers, Stroock and Varadhan completely solved this problem in the case where the coefficient $a_{i j}$ was uniformly elliptic, bounded and uniformly continuous, and $b_{i}$ was bounded and measurable. The papers were remarkable in so many different ways, giving us new and flexible probabilistic machinery that is still used routinely today, and at the same time demonstrating its worth by solving this basic problem in the analysis of PDEs.

Varadhan [18] had been exploring exponential martingales. Then, in the remarkable papers with Stroock, [11] [12], they assigned a Markov diffusion process to the elliptic PDE (1). They proved that in the case where $a$ is continuous and $b$ is bounded and measurable, then there is at most (and so exactly) one Markov process $X$ so that for each $T>0$ and each fixed $\theta$ in $\mathbb{R}^{d}$

$$
e^{\int_{T}^{t}\left(\theta \cdot d X_{t}-\frac{1}{2} \theta \cdot a \theta d t--\theta . b d t\right)}
$$

is a martingale. Everything seemed to slot into place. We were given a new technology of weak solutions to stochastic differential equations, and a completely novel way, based on using exponential martingales, to prove that such solutions are unique. At the same time the results of Tanaka and Krylov were extended to show the existence of the weak solution. Stroock and Varadhan moved away from a study of solutions to a PDE (which were obscure) to looking at the action of the operator (1) on an exponential function (which was a local calculation and straightforward) and used stochastic integral equations to characterise the Markov process. The proofs are deep and wide ranging; in their later book on the subject they exploit techniques from topics such as singular integrals to get the estimates they need.

One can easily widen the methodology to give good local notions of $L T h m$ 3.1), existence of solutions, and a maximum principle ([14] Thm 3.3) so solutions have the appropriate uniqueness. Viscosity-style solutions are not required for a good theory in this setting.

It seemed for many years that this intrinsic difficulty prevented these uniqueness results being extended to the uniformly elliptic bounded measurable case; but in 1997, in [8], Nadirashvili showed that the Stroock-Varadhan result is the best possible and gave the first counterexample to uniqueness in the bounded 
measurable case.

We emphasise that, although this is a result settling a key result in PDE theory, it, and the methodology developed around it, are important to probability. We should also not be surprised that probability seems the best route, and perhaps the only route, to studying these PDEs in depth. A Feller diffusion processes $\mathrm{X}_{t}$ is robustly associated to its transition semigroup defined on $C_{0}$ by

$$
P_{t}(f)(x):=\mathbb{E}\left(f\left(X_{t}\right) \mid X_{0}=x\right) .
$$

This semigroup characterises the diffusion, and its infinitesimal generator defines a closed operator $L$ on $C_{0}$. Without stochastic differential equations, any study of the process associated to it had to go in the reverse direction. Close $L$, use the fundamental solutions to the closed PDE to identify the semigroup, and use the semigroup to get the process. This is really quite problematic because PDEs locally characterise the behaviour of functions $f$. The beauty of the martingale characterisation of $X$ is that, in contrast to the semigroup description, it is also a local characterisation of $X$. With serious work and the skill of masters, this method would lead to the proof of appropriate uniqueness and existence statements for solutions to these PDEs and many other systems.

At the time of Stroock and Varadhan's work, I do not think it was at all obvious that the correct approach to proving tough questions about these PDEs was through probability. It was a tough journey they made. Now, of course, we realise that Markov diffusions are intrinsically more robust than their PDE counterparts and can be treated in a rigorous mathematical way across a wide context, including some (e.g. fractal environments and path spaces) where classical PDEs have little or no meaning.

\subsubsection{First-Order PDEs and Large Deviations}

It is a tautology to say that many of the most interesting features of our world and in our own personal experience evolve with time. Some evolve in a clear and predictable way. Others evolve in a less predictable and more random way. Some settle quickly to a large scale equilibrium, where the location and nature of that equilibrium is determined on a microscale by a steady incidence of rare events or large deviations. As a discipline, Stochastic Analysis is concerned with providing mathematical tools that are flexible enough to describe and study such systems, and at the same time concrete enough that they give real insight and lead one to address the real difficulties inherent in modelling them.

I would like to spend a little time discussing a beautiful paper [15] from 1966 where Varadhan is already using probabilistic methods to deeply understand a problem in non-linear first-order PDEs. The paper is fascinating for the way that, drawing on previous work of Donsker and Schilder, it forms a clear precursor result for the Large Deviations theory that would come later, and for the ease with which it brings together analytic tools that are familiar in finite-dimensional contexts (saddle point and steepest descent methods) and uses them in an infinite-dimensional setting, side-by-side with these emerging tools of Large Deviations, to create a decisive result. 
Donsker had shown that the solution $u_{\varepsilon}$ to the initial value problem

$$
u_{t}=u u_{x}+\frac{1}{2} \varepsilon^{2} u_{x x}+p(t, x)
$$

converges, as $\varepsilon \rightarrow 0$, to the solution to

$$
u_{t}=u u_{x}+p(t, x) .
$$

A key step was the Hopf transformation

$$
V(x):=\frac{1}{C} \exp \int^{x} \frac{-u(y)}{2 \kappa} d y
$$

which results in $V$ satisfying a third order PDE that factorises to the extent that it is obvious that if $V$ satisfies the linear equation

$$
V_{t}=\frac{1}{2} \varepsilon^{2} V_{x x}+\frac{1}{\varepsilon^{2}} p(t, x) V
$$

then $u$ satisfies the original equation. Donsker understood that (at least for each fixed $\varepsilon>0$ ) this linear equation has an easy probabilistic interpretation as an exponential path integral. Meanwhile, his student Schilder had, in his remarkable thesis, developed Laplace's method into a rigorous asymptotic expansion for certain families of exponential path integrals (M. Schilder, Some asymptotic formulas for Wiener integrals, Trans. Amer. Math Soc. 125 (1966), 63-85).

Varadhan took these results as his backcloth and put them together in a beautiful, clean and more abstract way, with the now famous formulation where one establishes a lower bound on open sets and upper bound on closed sets.

Using this more concise overview and further demonstrating his technical power by proving the necessary bounds for this particular problem, Varadhan established a much more comprehensive result than his predecessors.

Varadhan's result in that paper was to show that if $f$ is convex then the solution to the initial value problem

$$
u_{t}=[f(u)]_{x}+p(t, x)
$$

can be easily constructed or approximated by successive solutions to linear problems.

The paper is remarkable and influential. Laplace's method has been used in the study of tail behaviour for iid sums since Cramer, and now it was a nontrivial tool that could be effectively used in infinite-dimensional settingsand even applied to give insight into the behaviour and origin of a class of nonlinear PDEs.

The notes from Courant [18] set out the importance of using exponential martingales to study the behaviour of general diffusion processes. This is an idea that is obviously at the heart of the work on the martingale characterization of diffusion processes and its applications to the theory of PDEs with continuous coefficients - one of his major works. Meanwhile [19] is an early example of solving a PDE with homogenization of the coefficients. 


\section{PDEs and Probability in the 60s.}

At the time of this early work described in this section of my article, I do not think it was at all obvious that the correct approach to answering outstanding questions about these PDEs was through probability; there were other pioneers for sure: Itô and McKean for example. But I am sure it was a tough journey they all made and, as I have mentioned, the results and methodologies stood the test of time.

Forty years later, as we mentioned above, we now understand that Markov diffusions and these methodologies are intrinsically more robust and general than their PDE counterparts and can be treated and exploited in a rigorous mathematical way across a wide context.

The distinction between the large deviations of the two Markov processes with the same co-variance introduced in Section 0.4 vanishes in the diffusion/PDE limit, although it might be critically important to financial regulators.

Viewing macroscopic physical quantities as local integrals of functionals defined on spaces of paths is a deep and powerful concept.

Diffusions can exist in fractal like environments where there are no local charts and classical PDEs have no meaning. They are often given meaning through a theory of Dirichlet forms and weak formulations. Pollutants evolving though soil might be modelled by such methods. At the same time these methods again allow rigorous constructions of diffusions on nonlinear path spaces and lead to rigorous treatment of such things as operators and Sobolev structures on these spaces for the first time.

\subsection{The Support Theorem-Understanding the Itô Differential Equation}

\section{On Extending the Wong-Zakai Theorem}

One of the key results in [11] and [12] associates a unique diffusion process to any PDE of type (1), providing that $a$ is strictly positive as a quadratic form and continuous and $b$ is bounded and measurable.

If Stroock and Varadhan had assumed their equations had Lipschitz continuous coefficients then (as was remarked in [11]) Itô's theory would have given them all the information they would have needed.

A vector field $V$ on $\mathbb{R}^{d}$ defines, for each $x \in \mathbb{R}^{d}$, a vector or direction $V(x)=$ $\left(v_{1}(x), \ldots, v_{d}(x)\right)$ in $\mathbb{R}^{d}$. Vector fields are useful for describing how systems evolve. For example, one might look for paths $t \rightarrow \gamma_{t} \in \mathbb{R}^{d}$ that have velocity that matches the vector field or, in more mathematical language, solve the differential equation

$$
\dot{\gamma}_{t}=V\left(\gamma_{t}\right) .
$$

For Lipchitz vector fields there is always a unique solution with given starting point. In the example $V=(-y, x) \in \mathbb{R}^{2}$ the trajectories are circles centred at 
the origin. As we vary the initial state $\gamma_{0}$, the map from initial state to state at time $s$ defines an invertible map or flow $\pi_{s}$ by

$$
\pi_{s}\left(\gamma_{0}\right):=\gamma_{s} .
$$

In our example it rotates the plane by an angle $s$.

Now suppose that one places more interest on the interactions of the system $\gamma$ with other evolving systems $t$ and $\tau$. There are two very simple interactions, the first is replication where every increment of $\tau$ is replicated by an increment of $\gamma$

$$
d \gamma_{t}=d \tau_{t}
$$

and the second is the ordinary differential equation reflecting the effect of a vector field, which we now write as

$$
d \gamma_{t}=V\left(\gamma_{t}\right) d t
$$

for consistency.

A much richer range of interactions becomes immediately available if we mix these approaches. Suppose that $V^{0}, \ldots, V^{n}$ are a family of vector fields on $\mathbb{R}^{d}$ and that $\tau=\left(\tau_{1}, \ldots, \tau_{n}\right)$ is a (smooth enough) path in $\mathbb{R}^{n}$ then consider

$$
d \gamma_{t}=\sum_{j=0}^{n} V^{j}\left(\gamma_{t}\right) d \tau_{j, t}
$$

or

$$
d \gamma_{t}=V\left(\gamma_{t}\right) d \tau_{t}
$$

for short. It is helpful to view $\gamma$ as the response of the system to the control $\tau$; the map $\tau \mapsto \gamma$ is today known as the Itô map.

This equation includes the previous two via a judicious choice of $\tau$ and $V$. By adjoining variables the framework also includes the apparently more general case

$$
d \gamma_{t}=V\left(\gamma_{t}, \tau_{t}\right) d \tau_{t}
$$

through replicating the variable $\tau$ to $\tilde{\tau}$ and setting $\tilde{\gamma}=(\gamma, \tilde{\tau})$ :

$$
\begin{aligned}
d \tilde{\tau}_{t} & =d \tau_{t}, \quad \tilde{\tau}_{0}=\tau_{0} \\
d \gamma_{t} & =V\left(\gamma_{t}, \tilde{\tau}_{t}\right) d \tau_{t}
\end{aligned}
$$

is a differential equation of the prescribed type. In this way, geometers might note that one class of such systems is formed by the connections on a manifold. In this case, the path on the manifold is the control and the horizontal lift to the bundle is the response. Another class of examples appears in Cartan development - in this case the control is naturally the path in the Lie algebra and the response is the path developed onto the Lie group. 
In classical control theory the language is usually a little different. One can affect the evolution of a system by applying a control $\sum c_{j}(t) V^{j}$, mixing the different effects and considering the response

$$
d \gamma_{t}=\sum_{j=0}^{n} c_{j}(t) V^{j}\left(\gamma_{t}\right) d t
$$

In optimal control, the discussion usually constrains $\left(c_{j}(t)\right)$ to lie in some fixed compact convex set $K$ and optimises the choice to maximise some coordinate of the solution at the terminal time. By introducing

$$
C_{t}=\left(\int_{0}^{t} c_{1}(s) d s, \ldots, \int_{0}^{t} c_{n}(s) d s\right)
$$

one sees that the classical control theory problem is also easily articulated in this language

$$
d \gamma_{t}=V\left(\gamma_{t}\right) d C_{t}
$$

where the standard optimal control problem can then be seen to maximise some coordinate of the solution (the benefit) at the terminal time subject to a constraint (the cost) on the length of the path $C_{t}$ in an appropriate semi-norm and parametrises this control at unit speed.

To make sense of (2) requires some regularity between $V$ and $\tau$. If $V$ is Lipschitz and so is $t \rightarrow \tau_{t}$, then, by rewriting the equation in a form similar to (3), one can use the classical theory of ordinary differential equations to deduce the existence of unique solutions to the initial value problem and the existence of flows and so on. However, as the replication example makes clear, (2) should have meaning in some contexts where $\tau$ is not differentiable and there are no $c_{j}(t)$. It was the remarkable achievement of Itô, now taught in most major mathematics and engineering schools world-wide, to give this equation meaning as $\gamma$ ranges over almost every Brownian or semi-martingale path. Because Brownian motion $W_{t} \in \mathbb{R}^{n}$ has independent identically distributed increments with the correct homogeneity, it is at least intuitively obvious that the solutions to the Itô-Stratonovich equation

$$
d X_{t}=V\left(X_{t}\right) \circ d W_{t}+V^{0}\left(X_{t}\right) d t, \quad X_{0}=a
$$

would evolve in a way that was independent of their history, given their current position. That is to say $X_{t}$ is a Markov diffusion process. There are subtleties to get the exact theorems, but broadly it is easy to see from Itô's theory that if

$$
L u=\left(\sum_{j} v_{k}^{j} \frac{\partial}{\partial x_{k}} v_{l}^{j} \frac{\partial}{\partial x_{l}}+v_{k}^{0} \frac{\partial}{\partial x_{k}}\right) u
$$

and $f(x)$ is a bounded continuous function, then starting $X$ at $x$ at time $t$ and allowing $X$ to run until time $T$, and then evaluating $f$ at that location and averaging:

$$
u(x, t)=\mathbb{E}\left[f\left(X_{T}\right) \mid X_{t}=x\right]
$$


gives the solution to the boundary value problem

$$
\begin{aligned}
\frac{\partial u}{\partial t} & =L u \\
u(x, T) & =f(x)
\end{aligned}
$$

so expressing the solution $u$ as an integral of $f$ (Itô functional) over Wiener Space. One of the striking points is that the equation did not have to be elliptic or satisfy Hörmander's condition. As the example

$$
\begin{aligned}
V & =(1,0, \ldots, 0) \\
L & =\frac{\partial^{2}}{\partial x_{1} \partial x_{1}}
\end{aligned}
$$

shows clearly, even though $X$ diffuses it is not forced into any sort of spreading out in all directions; the diffusion, if it starts at a point, might well stay in a sub-manifold going through the starting point (a diffusive Hamiltonian system might still conserve momentum or energy). This explains the difficulty that one has in the subelliptic case in interpreting (4) in any naive way. If the process stays on a submanifold, e.g. on a circle centred on the origin, then the solutions will have no natural smoothness as one traverses from one sphere to adjacent ones. In general the curved nature of these surfaces means that $\frac{\partial^{2}}{\partial x_{i} \partial x_{j}} u$ will have no meaning, even though $u$ will — under reasonable hypotheses - be smooth on the circle. The fundamental solutions to (1) are evolving measures that, in general, do not have a density to Lebesgue measure that can be differentiated twice.

It is obviously an utterly basic question to understand, in terms of equation (1), the dependency of the solution at a point $\left(x_{0}, t_{0}\right)$ on this boundary data and identify the conservation laws. In probabilistic language: which sets support and contain the evolution? how does the evolution leave the domain?

"The support theorem" is the decisive answer, and today extends even to stochastic PDEs. It was motivated not only by the desire to extend Nirenberg's maximum principle to elliptic $L$ with continuous coefficients, but also to understand how to generalise Nirenberg's result to the degenerate setting where $a$ is only non-negative. The solution is a story of paths and control theory. The solution, in [14] and [13], is both intuitive, radical and technically deep. It is a story of a fundamental kind about approximation of solutions to stochastic differential equations by ordinary differential equations. The entire long last chapter of Ikeda and Watanabe's book (another ground breaking contribution to Stochastic Analysis) is dedicated to giving a second proof.

The ramifications of the methods have implications that go far beyond the fundamental problem solved. For example, the ideas underpin the standard construction of stochastic flows - a crucial concept for Bismut in [3] who gave a new proof of the index theorem.

One-Dimension of Rough Control Today, the first steps in understanding this work come from two later and, in some sense, deeply misleading papers, by 
0.6. THE SUPPORT THEOREM—UNDERSTANDING THE ITÔ DIFFERENTIAL EQUATION19

Doss and Sussman. Consider the controlled differential equation

$$
\begin{aligned}
d \tau_{t} & =V\left(\tau_{t}\right) d \gamma_{t}+V_{0}\left(\tau_{t}\right) d t \\
\tau_{0} & =a
\end{aligned}
$$

where $\gamma$ is a smooth real (i.e., in $\mathbb{R}^{n}, n=1$ ) valued path and $V, V_{0}$ are Lipschitz vector fields, then the path $\tau$ is uniquely defined using classical tools. Independently, each showed that the map $\gamma \mapsto \tau$ extends to all continuous $\gamma$ and is continuous in $\gamma$ in the uniform topology. It is clearly the unique extension with this property. We can thus give meaning to (5) for any continuous real valued path $\gamma$. In the case where $\gamma$ is a realisation of a 1-dimensional Brownian path, this definition almost surely coincides with the Itô-Stratonovich solution.

Now, if $\gamma_{t} \in \mathbb{R}$ is a continuous path for $t \in[0,1]$, and $\gamma_{t}^{(n)}$ is its dyadic piecewise linear approximation (i.e. $\gamma_{t}^{(n)}=\gamma_{t}$ if $t \in 2^{-n} \mathbb{Z} \cap[0,1], \gamma^{(n)}$ is continuous on $[0,1]$ and linear on each interval in $\left.[0,1] \backslash 2^{-n} \mathbb{Z}\right)$ then $\gamma^{(n)} \rightarrow \gamma$ uniformly. In particular, no matter how rough $\gamma$, if one defines $\tau^{(n)}$ to be the solution of (5) obtained by replacing the control $\gamma$ with its dyadic piecewise linear approximation $\gamma^{(n)}$ and solving the classical equation, then the $\tau^{(n)}$ converge to $\tau$. This gives a strong intuitive interpretation of (5) for rough one-dimensional $\gamma$. Restricting to the (Itô-Stratonovich) case where $\gamma$ is a randomly chosen Brownian path, one recovers the earlier result by Wong and Zakai that gave insight into the meaning and support of one-dimensional SDEs (stochastic differential equations).

Multiple Dimensions of Rough Control The results of Doss and Sussman are misleading - not because they are wrong, but because something rather miraculous happens if $\gamma$ takes its values in a one-dimensional space. In the multi-dimensional case it is simply and spectacularly false. The Itô functional $\gamma \mapsto \tau$

$$
d \tau_{t}=\sum_{i} V^{i}\left(\tau_{t}\right) d \gamma_{t}^{i}+V_{0}\left(\tau_{t}\right) d t, \quad \tau_{0}=a
$$

is not at all continuous in $\gamma$ in the uniform norm if the vector fields $V^{i}$ do not commute. It is not even closable in the space of continuous paths.

To get their support theorem, Stroock and Varadhan proved two conceptually fundamental results.

Stroock and Varadhan proved that if one approximates a Brownian path $X_{t}$ through its dyadic piecewise linear approximations $\gamma^{(n)}$ and solves the resulting classical ordinary differential equations then, almost surely, the $\tau_{t}^{(n)}$ again converge in law to the Itô-Stratonovich solution to the SDE driven by $X$. In this way they proved that the closed support of the law of $\tau_{t}$ is contained in the closure of the points where $\tau$ could reach when controlled by a piecewise smooth path $\gamma$.

Stroock and Varadhan also proved that if $\gamma$ was smooth, and if one conditions a Brownian path to be close to it in the uniform norm, then the solutions to the stochastic equation driven by the conditioned process converge to the solutions 
to the deterministic equation. From here one can see that the closed support of the $\tau_{t}$ controlled by piecewise smooth $\gamma$ is not bigger than the stochastic support.

From a more modern perspective, this extension of the Wong-Zakai theorem ${ }^{2}$ also follows from work of Sipilainen [10] who proved that the dyadic polygonal approximations to a Brownian path converge almost surely in the rough path metric.

The extension to Wong-Zakai, and the weak continuity theorem are far, far deeper results than the analogous one dimensional results. The difficulty of these results can be gauged by the fact that the statement that a path be close to $\gamma$ in the uniform norm on $[0,1]$ implicitly depended on a notion of distance in $\mathbb{R}^{n}$. Brownian motion conditioned to lie within $\varepsilon$ of $\gamma$ for one norm on $\mathbb{R}^{n}$ bears little statistical resemblence to the same process conditioned to lie within $\varepsilon$ of $\gamma$ for a second norm on $\mathbb{R}^{n}$, because these events correspond, are both rare and essentially distinct. The Stroock-Varadhan argument held for the box norm; later, Ikeda and Watanabe used the last chapter of their book to prove a similar result for the spherical norm. More recent work, presented in St Flour, allows the same result for any norm but depends on many more recent and deep developments, such as the boundary Harnack principle of Burdzy and Bass.

The conceptual content of these results is easy to take for granted today because it is so natural that the results should be true; they are basic and absorbed into our folklore. But they are also difficult. Had they not been true, then of course the whole subject would have developed differently. As an illustration of how the techniques go further than the basic results, we briefly discuss the existence of flows. Flows are solutions defined for every starting point $a$. Because there are uncountably many $a$, the Itô approach does not in itself allow such simultaneous solution. On the other hand the approximations to the solution using piecewise linear paths automatically provide flows for each approximation, and getting this sequence of diffeomorphisms to converge is possible and easier than trying to obtain the flow in other ways directly. Such flows were important to Bismut in his ground-breaking work on the Index theorem.

\subsection{The Donsker-Varadhan Theory of Large De- viations}

Introducing new mathematical abstraction can be dangerous and sometimes even an intellectual black hole. But it is also the life blood that allows mathematics to move forward and contribute to the wider world. At its most positive, one could think of "zero" - who needs a name for nothing? Closer to home, the introduction of filtrations of sigma algebras (adapted from measure theory) as a way of describing an evolving but partial knowledge of a system seems so dry that it could not have application.

\footnotetext{
${ }^{2}$ At around the same time, and independently, J.M.C. Clark also tackled this problem in his doctoral thesis
} 


\subsection{THE DONSKER-VARADHAN THEORY OF LARGE DEVIATIONS 21}

My own score chart is positive about abstraction when it leads to simplification of arguments, and the extension of existing results to a broader class of significant examples. I get very excited when the approach leads to to a completely new application. Successful innovations distinguish themselves from the black holes because they tend to be accompanied by an equally serious new approach which, although naturally proved in this level of abstraction, applies widely in settings where the abstract theory that justifies it is of little consequence. Leonardo Pisano brought us zero, but he also gave us Liber Abaci, brought the Hindu-Arabic algorithms of arithmetic to Europe, demonstrated the value of positional notation in denoting numbers, and made a non-trivial contribution to the development of (Merchant) Banking. Kolmogorov set out the axioms of probability in terms of measure theory, but immediately considered filtrations (sequences of nested $\sigma$-algebras), gave us a proper definition of independence and conditional expectation, and proved the strong law of large numbers in this context. The ideas are very abstract - it took me weeks to see any connection between the definitions and the intuitive concepts when I first came across the ideas. But the solid foundations it gave to probability, and the new theorems that came out of it, were a fundamental prerequisite to the massive development of Stochastic Analysis and probability as the toolset we have today.

In developing the theory of Large Deviations, Varadhan made a landmark achievement - he certainly proved hard theorems, demonstrating the value of the framework. Today, whether one is explaining the superconductivity associated to Josephson junctions, modelling stochastic resonance, managing communication networks, understanding the spectra of the large random matrices that occur in the analysis of mobile wireless communication channels, or understanding random surfaces, one gains substantial insight from Large Deviation theory. Rare events can be the crucial element in understanding the evolution of high dimensional (they happen) non-linear (their effects persist) systems.

\section{Short Time Behaviour-Geodesics}

Large Deviation theory provides the language and the basic results needed to describe and analyse these types of events, and even to model the systems when they are conditioned to have exceptional behaviour. In the papers [16] and [17] one can see the picture of large deviations theory being shaped as he proves a hard theorem. As before, Varadhan is proving theorems about non-constant coefficient elliptic and parabolic PDEs under relatively weak conditions on the coefficients. For example, consider $L$ where $a$ is Hölder and uniformly elliptic:

$$
L u=\sum_{j, k} a_{j k} \frac{\partial}{\partial x_{j}} \frac{\partial}{\partial x_{k}} u
$$

Then one can construct (Miranda, Friedman) a unique minimal solution on $\mathbb{R}^{d}$ :

$$
\frac{\partial}{\partial t} p_{\varepsilon}=\frac{1}{2} L p_{\varepsilon}
$$


which is positive $p_{t}(x, y)$ on $D$ and has boundary data $\delta_{x}$ at $t=0$.

In the customary way at that time, (Stroock and Varadhan came later) this is associated to a semi-group and a unique diffusion. Suppose that $D$ is open and that all boundary points are externally accessible; then we might be interested in the distribution of the time $T_{D}$ as the associated diffusion $X_{t}$, started at $x$, hits $\partial D$. From the Itô perspective it is easy to see that the Laplace transform of this random time solves the eigenfunction problem

$$
\begin{aligned}
\phi(x, \lambda) & :=\mathbb{E}_{x}\left[e^{\lambda T}\right] \\
L \phi & =\lambda \phi,\left.\quad \phi\right|_{\partial D}=1 .
\end{aligned}
$$

Varadhan gives a bare handed proof that

$$
\lim _{\lambda \rightarrow \infty}\left[-\frac{1}{\sqrt{2 \lambda}} \log \phi(x, \lambda)\right]=d(x, \partial D)
$$

and uses this to prove that the fundamental solution $p(t, x, y)$ has the property

$$
\lim _{t \searrow 0}-2 t \log p(t, x, y)=d(x, y)^{2}
$$

where the convergence is uniform on bounded regions. It is only for solutions defined on the whole of $\mathbb{R}^{d}$. This result is obvious if the coefficients are constant, but far deeper in this generality. It is the core technical bound that, when combined with the Large Deviations framework, gives a series of beautiful results in the second paper and provides a rich immediate justification for the framework.

The rigorous probability starts here. It was well known by the time the paper was written that the conditions on the operator $L$ were strong enough to associate to it a unique strong Markov diffusion $\left(X_{t}\right)_{t \in[0, T]}$ whose Feller transition semigroup was $p_{t}(x, y)$. We can use this reference process to define a family of measures on $C[0, T]$ by rescaling time

$$
\mathbb{P}_{x}^{\varepsilon}(A)=\mathbb{P}_{x}\left(\left.X_{\varepsilon t}\right|_{t \in[0, T]} \in A\right) .
$$

We can fix $A$ and consider in general the behaviour as $\varepsilon \rightarrow 0$ of $\mathbb{P}_{x}^{\varepsilon}(A)$. Note that $A$ is a generic set of paths and so this is truly an infinite-dimensional question. If the set of paths $A$ is separated, in the uniform metric, from the path that stays at $x$ for all time, then the probability that the reparameterised path $\left.X_{\varepsilon t}\right|_{t \in[0, T]}$ started at $x$ is in $A$ becomes increasingly unlikely as $\varepsilon \rightarrow 0$. In fact one can often get a really quite precise asymptotic picture. Suppose that $x \in D$, an open set in $\mathbb{R}^{d}$, and $A_{D}$ is the set of paths $\gamma$ in $C\left([0, T], \mathbb{R}^{d}\right)$ that remain in $D$ until at least time $T$ and which, at time $T$, are in a ball of radius $\delta>0$ centred on $y \neq x$

$$
A_{D}=\{\gamma \mid \gamma([0, T]) \subset D, d(\gamma(T), y)<\delta\}
$$

The set $A_{D}$ is open in the uniform topology and, because $y \neq x$, is separated from the trivial path at $x$. It is probably best to give an example. 


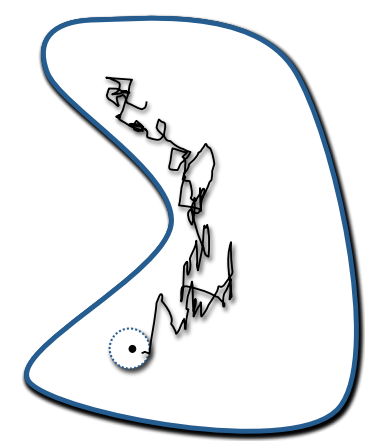

Can one be more precise? For example, how does the probability change if we change the shape of the region $D$ (in particular if we let it be the whole of $\left.\mathbb{R}^{d}\right)$ ? How does it depend on the diffusion coefficients $a$ that determine $L$ and $X ?$

We have already mentioned that, although rare events are rare, if there are several of them then it is very unusual that they are equally rare. There is an intuition that, if we aggregate a family of rare events $A_{i}$ into a single rare event $\cup A_{i}$, one of the rare events in the aggregate will be much less rare than the others, and so dominate and determine the rareness of the aggregate. This would certainly be true if $\mathbb{P}_{\varepsilon}\left(A_{i}\right)=r_{i} e^{s_{i} / \varepsilon}$; if $r_{j}>0$ and the $s_{j}$ are distinct then

$$
\max _{j<N} \lim _{\varepsilon \rightarrow 0} \frac{\sum_{i<N} r_{i} e^{s_{i} / \varepsilon}}{r_{j} e^{s_{j} / \varepsilon}}=1 .
$$

At the heart of any Large Deviations argument one has to prove that an appropriate extension of this relationship holds in a setting where the $i$ range over a continuum instead of a finite set.

Varadhan introduced a functional on paths $\gamma$ that would determine their local rareness for the diffusion process over short times. It now has a natural feel to it as the energy of the path

$$
I(\gamma):=\frac{1}{2} \int_{0}^{T} \dot{\gamma}_{t} a\left(\gamma_{t}\right)^{-1} \dot{\gamma}_{t} d t
$$

(or $+\infty$ where $\dot{\gamma}$ is not defined almost everywhere) determined by the diffusion 
coefficient $a$. He could then use his estimate of the probability of the reparameterised process being close to $\gamma$ to prove the following result.

Theorem Let $G \subset C\left([0, T], \mathbb{R}^{d}\right)$ be open and $C \subset C\left([0, T], \mathbb{R}^{d}\right)$ be closed. Then

$$
\begin{aligned}
& \limsup _{\varepsilon \rightarrow 0} \log \mathbb{P}_{x}^{\varepsilon}[C] \leq-\inf _{\gamma \in C} I(\gamma), \\
& \liminf _{\varepsilon \rightarrow 0} \varepsilon \log \mathbb{P}_{x}^{\varepsilon}[G] \geq-\inf _{\gamma \in C} I(\gamma) .
\end{aligned}
$$

In this way Varadhan established asymptotic lower bounds on the probability that a random path $X_{\varepsilon t}$, run over the interval $[0, T]$, would be in a given open set of paths. At the same time he had an asymptotic upper bound on the equivalent inclusion for closed sets of paths. Varadhan also allowed the sets $G$ and $C$, on the left hand side, to vary.

We can now look again at the example of the paths that stay in the domain $D$. As $\varepsilon \rightarrow 0$, almost all paths from $x$ to $y$ will stay close to a minimising geodesic if there is one. There is an obvious phase transition according to whether $A_{D}$ contains the shortest path (in the $a$ metric) from $x$ to the ball centred on $y$. In our picture it does not.

Varadhan made this all precise and, in a beautiful application, related the fundamental solutions $p_{D}, p$ to the PDE restricted to the domain and on the whole of $\mathbb{R}^{d}$. An immediate consequence of Theorem 4.9 in that paper is that if $p_{D}$ is the fundamental solution for the equation restricted to a domain $D$ with Dirichlet boundary conditions then

$$
\lim _{t \searrow 0} \frac{p(t, x, y)}{p_{D}(t, x, y)}=1
$$

for every pair $(x, y) \in D \times D$ if and only if $D$ is convex in the Riemannian sense for the metric $a^{-1}$. This is a very fine remark which is considerably more delicate than the basic large deviations result above. It symbolises how, even in 1967, Varadhan had very serious examples to test the technology and perfect the theory of Large Deviations as well as beautiful applications.

\section{Long Time Behaviour-Occupation Measures}

Suppose a visitor to a finite neighbourhood is forced by his job to move around the neighbourhood in a Markovian way. As he moves around he interacts with the local inhabitants and would like to make friends. But they are cautious and only make friends if the visitor spends a considerable amount of time at their site and gives them significantly more than average attention. On average the visitor will spend equal time at each site, but even over long time intervals then time will not exactly equilibrate. How many friends can the visitor expect to make? How many good friends?

Mathematically, the problem is to look at a recurrent diffusion over long time periods. For example $X$ might be a Brownian motion on a compact multidimensional manifold. Then, from the ergodic theorem, one knows that for 
almost every $\omega$ the empirical occupation measure $\mu_{(T, \omega)}$ defined by

$$
\mu_{(T, \omega)}(f):=\frac{1}{T} \int_{0}^{T} f\left(X_{t}(\omega)\right) d t
$$

will converge to the normalised volume measure. But in general it will never get there. One would like to describe the fluctuations and exceptional behaviour of this random(ly evolving) measure.

I suppose (but do not know) that the theory really became confirmed when, with Donsker [4, 6, 7], Varadhan looked at this second intrinsically important and clearly infinite-dimensional problem of convergence. Again, the dimensionality and the specificness of the problem ensured that there were hard issues to be resolved, but the Large Deviations framework pointed out the direction and focused the considerable effort required allowing much better results than a more ad hoc approach.

They identified a rate function

$$
I(\mu)=\inf _{\substack{u>0 \\ u \in \mathcal{D}(L)}} \int_{x \in M} \frac{L u}{u}(x) \mu(d x)
$$

and established the Large Deviation principle, so providing a powerful family of estimates for exceptional behaviour of the occupation measure and its deviations from the invariant measure. This gives insight into what happens when one gets a large fluctuation and gives valuable information on the dynamics of the system conditional on this exceptional behaviour by minimising $I(\mu)$, subject to the constraint of the exceptional behaviour for $\mu$.

In other words, if we condition on our traveller making an unusually large number of friends, then we can expect and predict a lot of structure in how these contacts are built up. This work on occupation measures is highly applicable and recaptures Kac's use of exponential functionals to capture solutions to PDEs. As before there is always at least one basic concrete result that is needed to make it all work (no free lunch!). In this case one could cite [5], which proves under appropriate hypotheses that

$$
\lim _{t \rightarrow \infty} \frac{1}{t} \log \mathbb{E}_{x}\left[e^{-t \Phi(\hat{\mu})}\right]=-\inf _{\mu} \frac{1}{8}\left(\int\left|\nabla\left(\log \frac{d \mu}{d \nu}\right)\right|^{2} d \mu+\Phi(\mu)\right)
$$

This is determined using the diffusion metric, where $\nu$ is the invariant measure, $\hat{\mu}$ is the normalised general random occupation measure, and $\Phi$ is a lsc functional on probability measures with compact sets $\Phi \leq \lambda$.

The ramifications of the methodology established in these early papers have spread so widely that it would be impossible to do the whole subject any sort of credit and in any case others are better equipped to do this than this author. 


\subsection{Hydrodynamical Limits, Interacting Parti- cles and Other Questions}

We have seen that large scale non-linear phenomena can arise because of the influence of large deviations. The effect is exactly a balanced trade off between the rareness of the event, the magnitude of the event, and the influence it has on the system as a whole.

However, one needs caution. For the fact that large deviations matter throws doubt onto the framework in which they are studied, on the non-linear PDEs they justify, and on modelling in general. Most real world models have a granularity, but, like the simple random walk, sometimes there are central limit type theorems, and they can be modelled very effectively by Markov diffusion processes. However, as the introductory section in this article shows, the large deviations of the granular process may be very different to those of the limiting Markov diffusion. The macroscopic large deviations of the granular process are a function of the microstructure of the process. Microstructure that is lost on taking the diffusion limit. This is important, and also a challenge - one cannot interchange orders of limits without justification.

So, there is a very real challenge to take reasonable microscopic models of non-linear physical systems (such as fluids), systems where the non-linear and large deviation effects are likely to be highly relevant, and prove that the large scale behaviour can be successfully modelled (with PDEs or by other means). This is a major programme, proving there is a fluid limit for models with nontrivial microstructure, of current interest. Varadhan, with younger colleagues and students Kipnis, Ola (Rome), Quastel (Toronto) and H.T. Yau (Harvard) did as much as anyone to initiate this very important programme.

Almost by definition, the current state of understanding on the hydrodynamical limits and on particle systems has a huge amount of detail, and would require another long chapter to do it justice. Moreover, and sadly, I do not have enough expertise to summarise it accurately.

\section{Homogenised Hamilton-Jacobi Equations}

Sometimes homogenisation works, you can forget local structure, and central limit phenomena dominate! In this case, one might replace the rapidly fluctuating coefficients in the basic diffusion with a much more smoothly varying model, but still have a diffusion which, on the appropriate scales, was indistinguishable from the original. Varadhan had early work on this homogenising the coefficients of PDEs. He was also a co-author of the very influential unpublished - but widely circulated and referenced - contribution on homogenisation of the Hamilton-Jacobi equation, with Lions and Papanicolou which provided the weak viscosity solutions to the HJE equation that underpin recent work on the construction of Mather invariant sets, reconnecting PDEs and Lagrangian dynamics.

The impact of this work persists to the current time. KAM theory provides a suitable framework for understanding the dynamics of attracting sets for a sys- 
tem subject to a small periodic potential. Aubry and Mather, and then Mather alone, constructed invariant sets in the non-KAM non-perturbative framework. The work of Fathi and Evans, using weak viscosity solutions to the HJE developed in the seminal work with Lions and Papanicolou, constructs Mather invariants in the non-KAM non-perturbative framework.

\subsection{Conclusion}

The Fields Medals at Madrid illustrate how probability has come of age. Okounkov used random surfaces to connect work of Gromov and Witten with that of Donaldson and Thomas. Werner's work is motivated by statistical physics and links the evolving geometry of two-dimensional Brownian motion to conformal field theory. Tao used probabilistic motivations for many aspects of his plenary lecture.

It is inconceivable that Okounkov and Werner have not been substantially, even subconsciously, influenced by the work of Varadhan and his collaborators. Varadhan's work has influenced applications at the same level and his results and perspectives infuse over a wide horizon. Much of my work would have little interest without his contributions.

Probabilists around the world were delighted when Varadhan was awarded the Abel Prize; his deep and decisive contributions to this significant and young area of mathematics ensure that the Abel prize will get distinction from choosing Varadhan!

The author of this short article would like to offer his thanks to Professor Varadhan for his inspiration, to the Abel Foundation for their patience, and to the reader for getting this far. At the same time as he hopes to have given a flavour of some of Varadhan's work, he is very conscious of, almost by the same token, the simplifications and omissions that have distorted the accuracy of the presentation; for this he asks the readers' forgiveness. 


\section{Bibliography}

[1] M.T. Barlow. Heat kernels and sets with fractal structure. Contemp. Math. 338:11-40 (2003)

[2] M.T. Barlow, R.F. Bass, T. Kumagai, and A. Teplyaev. Uniqueness of Brownian motion on Sierpinski carpets. arXiv:0812.1802v1, 2008.

[3] Jean-Michel Bismut. Mécanique aléatoire, volume 866 of Lecture Notes in Mathematics. Springer-Verlag, Berlin, 1981. With an English summary.

[4] M. D. Donsker and S. R. S. Varadhan. Asymptotic evaluation of certain Markov process expectations for large time. I. II. Comm. Pure Appl. Math., 28:1-47; ibid. 28 (1975), 279-301, 1975.

[5] M. D. Donsker and S. R. S. Varadhan. Asymptotic evaluation of certain Wiener integrals for large time. In Functional integration and its applications (Proc. Internat. Conf., London, 1974), pages 15-33. Clarendon Press, Oxford, 1975.

[6] M. D. Donsker and S. R. S. Varadhan. Asymptotic evaluation of certain Markov process expectations for large time. III. Comm. Pure Appl. Math., 29(4):389-461, 1976.

[7] M. D. Donsker and S. R. S. Varadhan. Asymptotic evaluation of certain Markov process expectations for large time. IV. Comm. Pure Appl. Math., 36(2):183-212, 1983.

[8] Nikolai Nadirashvili. Nonuniqueness in the martingale problem and the Dirichlet problem for uniformly elliptic operators. Ann. Scuola Norm. Sup. Pisa Cl. Sci. (4), 24(3):537-549, 1997.

[9] Louis Nirenberg. A strong maximum principle for parabolic equations. Comm. Pure Appl. Math., 6:167-177, 1953.

[10] E.-M. Sipilainen. A pathwise view of solutions of stochastic differential equations. PhD thesis, University of Edinburgh, 1993.

[11] Daniel W. Stroock and S. R. S. Varadhan. Diffusion processes with continuous coefficients. I. Comm. Pure Appl. Math., 22:345-400, 1969. 
[12] Daniel W. Stroock and S. R. S. Varadhan. Diffusion processes with continuous coefficients. II. Comm. Pure Appl. Math., 22:479-530, 1969.

[13] Daniel W. Stroock and S. R. S. Varadhan. Diffusion processes with boundary conditions. Comm. Pure Appl. Math., 24:147-225, 1971.

[14] Daniel W. Stroock and S. R. S. Varadhan. On the support of diffusion processes with applications to the strong maximum principle. In Proceedings of the Sixth Berkeley Symposium on Mathematical Statistics and Probability (Univ. California, Berkeley, Calif., 1970/1971), Vol. III: Probability theory, pages 333-359, Berkeley, Calif., 1972. Univ. California Press.

[15] S. R. S. Varadhan. Asymptotic probabilities and differential equations. Comm. Pure Appl. Math., 19:261-286, 1966.

[16] S. R. S. Varadhan. Diffusion processes in a small time interval. Comm. Pure Appl. Math., 20:659-685, 1967.

[17] S. R. S. Varadhan. On the behavior of the fundamental solution of the heat equation with variable coefficients. Comm. Pure Appl. Math., 20:431-455, 1967.

[18] S. R. S. Varadhan. Stochastic processes. Notes based on a course given at New York University during the year 1967/68. Courant Institute of Mathematical Sciences New York University, New York, 1968.

[19] S. R. S. Varadhan. Boundary value problems with rapidly oscillating random coefficients. In Random fields, Vol. I, II (Esztergom, 1979), volume 27 of Colloq. Math. Soc. János Bolyai, pages 835-873. North-Holland, Amsterdam, 1981. 